\title{
The Optimization Decision Model of Sub-contractor Selection in Multiple Subproject and Its Solving Method of Genetic Algorithm
}

\author{
Huiyang Xie
}

\begin{abstract}
Along with the prosperity of market economy, the improvement of comprehensive the corporate strength, large and medium-sized projects become more and more. On the other hand, the tendering and bidding market has become increasingly fierce, the benefit of the enterprises who win the bidding generally cannot be too high. According to the main evaluation factors in the evaluation of bid, this paper established a quantitative evaluation index system, using the quantitative indicators as variables, built up the integer programming bid evaluation optimization decision model with comparative benefit maximization of competitive bid unit as the objective function and the general requirements of umbrella project to subproject as constraint conditions. According to the characteristics of the problem of sub-contractor selection in multiple subprojects, this paper put forward the corresponding algorithm of genetic algorithm. Examples show that the algorithm is effective.
\end{abstract}

Index Terms-Genetic algorithm, multiple subproject, optimization, sub-contractor.

\section{INTRODUCTION}

Along with the prosperity of market economy, the improvement of comprehensive the corporate strength, large and medium -sized projects become more and more. On the other hand, the tendering and bidding market has become increasingly fierce. If the winning enterprises adopt self-supporting construction, they will invest a lot of equipment and personnel, which resulted in increased cost expenses and difficult to control the construction cost. Aimed at the characteristics of each project, before the construction can be clearly for some project implement process of the subcontract, such already can reduce the enterprise investment, guarantee the interests of the enterprise, and can meet the requirements of the main contract in engineering quality and the limiting time. Therefore, how to select the subcontractor is a worthy of serious research topic.

In literature [1], the authors established the constraint conditions of $0-1$ programming evaluation optimization decision model, and used the Excel "planning" function to solve the model. The model in [1] can also use 0-1 programming problem of the implicit enumeration methods or branch and bound method to solve. But in this model, the number of variables is $\sum_{i=1}^{n} m_{i}$, when it meets the requirements

Manuscript received November 4, 2014; revised January 6, 2015. This research was financially supported by the National Science Foundation under grand 61370193.

Huiyang Xie is with Beijing Forestry University, 100083, Beijing, China (e-mail: xhyang@bjfu.edu.cn). of the bidding enterprises selected contractors more $\left(m_{i}\right.$ large), using the method of the 0-1 programming, such as implicit enumeration method, the branch and bound method, the efficiency of solving the problems is very low.

In recent years, intelligent algorithm represented by GA, $\mathrm{PSO}$, etc are increasingly used in various combinations of engineering optimization problems [2]-[5], and has obtained many achievements. According to the problem of sub-contractor selection in multiple subprojects, if we adopt the article [1] model, the intelligent method based on the population can use binary encoding way of modeling. When the bid unit of each subproject is large, the code can lead to the length of population Individual too long, and affect the efficiency of algorithm search.

Aiming at the problems above, the use of integer variables create a multiple sub-subcontractor selection mathematical models, a new mathematical model objective function and constraints more concise, and easy to calculate. However, the variables in the model appear in the subscript, classical algorithm cannot be used to solve optimization problems. This paper uses genetic algorithms to solve the problems.

\section{THE SUB-PROJECT EVALUATION INDEX SYSTEM AND EVALUATION FUNCTION}

Generally speaking, the project is always through tendering to select the contractor, which are mainly public tender, Two tender and international tenders. They are normally divided into four stages, namely: the tender preparation phase, bid preparation stage, the opening stage of evaluation and decision about the label phase. Among them, the opening bid is the core part of the whole stage bidding activities; the main square in the tender is to make the greatest relative benefit principle, a good selection of subcontractors. Quantitative methods to identify key subcontractors bid is scientific and reasonable to establish an effective evaluation mathematical model, each qualitative indicators into quantitative indicators to minimize the influence of subjective factors, so as to promote scientific decision-making throughout the bidding process democratization and rationalization.

In the evaluation, in addition to considering the tender offer bid beyond the enterprise, but also the duration of the project, construction quality, construction plan, the main material usage, construction experience and the reputation of a comprehensive evaluation. For the quantitative analysis of the evaluation process, we need a certain amount of quantitative indicators, quantitative evaluation factors of the individual [6], the paper gives an evaluation index system for the project 
as shown in Table I.

The principle of bid evaluation optimization decision model is that when choosing a contractor, starting from the bid invitation unit interests, making the bid invitation unit in the choice to the appropriate contracting units, obtaining the maximum relative benefit. Relative benefit here refers to the differential income the bid invitation unit acquired by comparing the contracting units including the index of project construction period, project quoted price etc. and the base price, pre-tender construction period of the bid invitation unit. According to the quantitative evaluation index system, relative income can be expressed in specific formula as follows: relative benefit $=\sum_{k=1}^{6} p_{k} \times(k$ th index of quotation $-k$ th index of bid $)$

$p_{k}$ is the conversion rate of return for $k$ th index, aimed to put the different units in objective function into ten thousand Yuan. Such as the conversion rate of return of project construction period can be identified as the economic and social benefits by shortening construction time each month. The conversion rate of return of the rate of excellent engineering can be identified as the wealth of intangible assets represented by every one percent of the rate of excellent engineering. Others are in a similar way.

TABLE I: ENGINEERING PROJECT EVALUATION INDEX SYSTEM

\begin{tabular}{|c|c|c|c|c|c|}
\hline$K$ & Factor of evaluation & Quantitative index & Units & Computational formula & Conversion rate of interest \\
\hline 1 & Tender offer & Tender offer & 10,000 & - & $P_{1}$ \\
\hline 2 & Time limit for a project & Time limit for a project & Month & - & $P_{2}$ \\
\hline 3 & Construction quality & Construction quality & $\%$ & Number of excellent works/total & $P_{3}$ \\
\hline 4 & Main material usage & Main material usage & 10,000 & - & $P_{4}$ \\
\hline 5 & Construction experience & Experience in similar projects & $\%$ & Number of similar projects/total & $P_{5}$ \\
\hline 6 & Corporate reputation & Contract completion & $\%$ & Number of completed works/total & $P_{6}$ \\
\hline
\end{tabular}

\section{Optimization MODEl OF SUB-CONTRACTOR SELECTION IN MULTIPLE SUBPROJECT}

For a single project, through bidding prequalification, they can select in accordance with the conditions of enterprises contracting units. At this time all costs, the technical parameters etc. in the book of contractors bidding which accord with the primary condition are listed are brought into the calculation formula (1) of the relative gains. Then compare their size. The tendered with the maximum relative income is the winning bidder. But for the same project of multiple subprojects bidding at the same time, each subproject has a number of bidding units with different time limit for a project, and the subproject of the mutual influence between the indexes. Therefore, the solution using the separate subproject bidding may not be necessarily the optimal solution of the entire project. At this time, we can establish the 0-1 programming model to solve [1]:

Suppose there are $\mathrm{n}$ subprojects bidding, the $i$ th subproject has $m_{i}$ units participated in the bidding. The mark of $i$ th subproject is as shown in Table II.

TABLE II: THE MARK OF RELATED PARAMETERS

\begin{tabular}{lllll}
\hline \hline Factor of evaluation & $\begin{array}{l}J \text { th bid } \\
\text { data }\end{array}$ & $\begin{array}{l}\text { Base } \\
\text { price }\end{array}$ & Units & $\begin{array}{l}\text { Conversion } \\
\text { rate of interest }\end{array}$ \\
\hline $\begin{array}{l}\text { Sub-project prices } \\
\begin{array}{l}\text { Time limit for a } \\
\text { project }\end{array}\end{array}$ & $S_{1 i j}$ & $\tilde{S}_{1 i}$ & Yuan & $P_{1 i}$ \\
$\begin{array}{l}\text { Construction quality } \\
S_{3 i j}\end{array}$ & $\tilde{S}_{3 i}$ & Month & $P_{2 i}$ \\
$\begin{array}{l}\text { Main material usage } \\
\text { Construction }\end{array}$ & $S_{4 i j}$ & $\tilde{S}_{4 i}$ & Yuan & $P_{4 i}$ \\
experience & $S_{5 i j}$ & $\tilde{S}_{5 i}$ & $\%$ & $P_{5 i}$ \\
Corporate reputation & $S_{6 i j}$ & $\tilde{S}_{6 i}$ & $\%$ & $P_{6 i}$ \\
\hline \hline
\end{tabular}

If the $i$ th subproject chooses the $j$ th contractor, then relative efficiency is:

$$
c_{i j}=\sum_{k=1}^{6} p_{k i}\left(s_{k i j}-\tilde{s}_{k i}\right)
$$

Introducing 0-1 variable, can establish a 0-1 programming model. And we can use the $0-1$ programming problem of the implicit enumeration methods, branch and bound method to solve. But in this model, the number of variables is $\sum_{i=1}^{n} m_{i}$, when it meets the requirements of the bidding enterprises selected contractors more ( $m_{i}$ large), using the method of the 0-1 programming, such as implicit enumeration method, the branch and bound method, the efficiency of solving the problems is very low.

Aiming at the problems above, the uses of integer variables create a multiple sub-subcontractor selection mathematical models.

Assuming that the $i$ th project subcontracted to the $x_{i}$ th subcontractor, then the new multi subproject bidding decision model of the objective function is:

$$
\max Z=\sum_{i=1}^{n} c_{i x_{i}}
$$

Variable is limited as:

$$
x_{i} \text { is an integer in }\left[1, m_{i}\right]
$$

Constraint conditions of each index in turn is:

1) The limit of the total price :

$$
M_{11} \leq \sum_{i=1}^{n} s_{1 i x_{i}} \leq M_{12}
$$

where $M_{11}$ is the lowest total price, $M_{12}$ is the highest total price. 
2) The limit of the total time :

$$
(1-a) M_{2} \leq \sum_{i=1}^{n} s_{2 i x_{i}} \leq(1+a) M_{2}
$$

where $M_{2}$ is the total time limit for a project quotation, Constant a is the ratio of floating up and down.

3) The limit of the excellent rate in contractor's total engineering:

$$
\sum_{i=1}^{n} \omega_{i 3} s_{3 i x_{i}} \geq M_{3}
$$

where $M_{3}$ is a fixed constant, $\omega_{13}, \omega_{23}, \cdots, \omega_{n 3} \geq 0$ is the weight coefficient. $\sum_{i=1}^{n} \omega_{i 3}=1$, They reflect the degree of importance on the quality requirements for each subproject in the whole project.

4) The limit of the main material in relative dosage :

$$
\sum_{i=1}^{n} \omega_{i 4} \frac{s_{4 i x_{i}}}{S_{1 i x_{i}}} \geq M_{4}
$$

where $M_{4}$ is a fixed constant, $\omega_{14}, \omega_{24}, \cdots, \omega_{n 4} \geq 0$ is the weight coefficient. $\sum_{i=1}^{n} \omega_{i 4}=1$. They reflect the degree of importance on the main material dosage requirements for each subproject in the whole project.

5) The limit of the experience rate in similar project:

$$
\sum_{i=1}^{n} \omega_{i 5} s_{5 i x_{i}} \geq M_{5}
$$

where $M_{5}$ is a fixed constant, $\omega_{15}, \omega_{25}, \cdots, \omega_{n 5} \geq 0$ is the weight coefficient. $\sum_{i=1}^{n} \omega_{i 5}=1$, . They reflect the degree of importance on the experience rate requirements in similar project for each subproject in the whole project.

6) The limit of the completion rate in contract:

$$
\sum_{i=1}^{n} \omega_{i 6} s_{6 i x_{i}} \geq M_{6}
$$

where $M_{6}$ is a fixed constant, $\omega_{16}, \omega_{26}, \cdots, \omega_{n 6} \geq 0$ is the weight coefficient. $\sum_{i=1}^{n} \omega_{i 6}=1$. They reflect the degree of importance on the completion rate in contract project for each subproject in the whole project.

In the above model, the decision variables appear in the left side of the subscript in the constraint (5) (10). The problems cannot be solved by the classical optimization algorithms. This paper adopts genetic algorithm to solve the problem.

For the constrained optimization problem, by using the penalty function method, the problem can be transformed into the following constrained optimization problems:

$$
\begin{aligned}
& \min f(x)=\sum_{i=1}^{n} c_{i x_{i}}-M\left[\alpha_{1} \max \left(M_{11}-\sum_{i=1}^{n} s_{1 i x_{i}}, \sum_{i=1}^{n} s_{1 i x_{i}}-M_{12}, 0\right)\right. \\
& +\alpha_{2} \max \left((1-a) M_{2}-\sum_{i=1}^{n} s_{2 i x_{i}}, \sum_{i=1}^{n} s_{2 i x_{i}}\right. \\
& \left.-(1+a) M_{2}, 0\right)+\alpha_{3} \max \left(M_{3}-\sum_{i=1}^{n} \omega_{i 3} s_{3 i x_{i}}, 0\right) \\
& +\alpha_{4} \max \left(M_{4}-\sum_{i=1}^{n} \omega_{i 4} \frac{s_{4 x_{i}}}{s_{1 i x_{i}}}, 0\right)+\alpha_{5} \max \left(M_{5}-\sum_{i=1}^{n} \omega_{i 5} s_{5 x_{i}}, 0\right) \\
& \left.+\alpha_{6} \max \left(M_{6}-\sum_{i=1}^{n} \omega_{i 6} s_{6 i x_{i}}, 0\right)\right] \\
& \text { s.t. } x_{i} \text { is an integer in }\left[1, m_{i}\right] \quad i=1,2, \cdots, n
\end{aligned}
$$

where $\alpha_{j}$ can be 0 or 1 , if they put forward on the $j$ th index in the above sixth index, then $\alpha_{j}=1$, Otherwise $\alpha_{j}=0$. In actual calculations, if we don't limit to an index, the item can be removed from the expression to simplify the calculation.

\section{THE GENETIC AlgORITHM FOR THE OPTIMIZATION MODEl OF SUb-CONTRACTOR SELECTION IN MULTIPLE SUBPROJECT}

\section{A. The Encoding Selection}

For the genetic algorithm of this problem, this paper adopts the natural number coding. Let the chromosome $x=\left(x_{1}, x_{2}, \cdots, x_{n}\right)$, where $x_{i}$ is an integer between 1 and $m_{i}$, said the $i$ project choose the $x_{i}$ th subcontractor of the $i$ th project.

\section{B. The Operation of Genetic Evolution}

\section{1) Interlace operation}

In this paper, we use the two point crossover operation which is a classic operation in genetic algorithm. We randomly select two tangent point $\mathrm{k}$, and exchange the middle part between the two parent individuals to produce offspring. By observing the double tangent point crossover of the integer coding we can know, if the parent satisfies the variable constraints (13), the resulting offspring will be the code which satisfies the variable constraints (13).

\section{2) Variation}

Bit value variation is for every positions $k_{1}, k_{2}, \cdots, k_{s} \quad\left(1 \leq k_{1}<k_{2}<\cdots<k_{s} \leq n, 1 \leq s<n\right) \quad$ in its gene $x=\left(x_{1}, \cdots, x_{k}, x_{k+1}, \cdots, x_{l}, x_{l+1} \cdots, x_{n}\right)$, let them be the following variation:

$$
\tilde{x}_{k_{i}}=t_{k_{i}}
$$

where $t_{k_{i}}$ is a selected number in $\left\{1,2, \ldots, m_{i}\right\} \backslash\left\{x_{i}\right\}$ with equal probability. During iteration algorithm, the integer mutation operation is performed with the probability of $p_{m}$.

\section{3) Calibration method of the fitness}

In this paper, we use the dynamic linear calibration method. This is the most commonly used calibration method. It can 
be expressed as follows:

$$
F=f-f_{\min }^{k}+\xi^{k}
$$

where superscript $k$ is the number of iterations. It shows that the parameter changes along with the increase in the number of iterations.

\section{4) Selection strategy}

This paper uses the proportional selection strategy. That is, the probability of each individual selected to genetic operation is the proportion between the fitness of individual and the sum of all fitness.

\section{Algorithm Process}

Step 1: Produce the initial parent population randomly $A_{1}$. $k=1$.

Step 2: Fitness computation.

Step 3: Do crossover operation with the $k$ th parent population $A_{k}$, get the population $B_{k}$.

Step 4: Do mutation operation with the $k$ th parent population $A_{k}$, get the population $C_{k}$.

Step 5: population regeneration.

Step 6: Determine whether a termination condition is satisfied? If satisfied, end the algorithm; if not, let $k \leftarrow k+1$, go to Step 3.

The main parameters of the algorithm as follows: the initial population size is 30 . The probability of crossover operator is $p_{c}=0.5$, the probability of mutation operator is $p_{m}=0.1$. The reserved number of elite between different generations is $z_{1}=5$. The number of tournament selection group is $z_{2}=5$. The max generation is $G=30$.

For the example in [1], using the algorithm proposed in this paper, producing the initial parent population randomly, we can get the optimal solution of the problem through the calculation of 20 times. The results are consistent with the results by other methods. It shows that the algorithm proposed in this paper is effective.

\section{CONCLUSION}

Aiming at the problem of sub-contractor selection in multiple subprojects, an optimization decision model based on the number of sub-contractor and relative income was introduced in this paper. The advantages are as follows:

1) The number of variables in model is $n$, which is far less than the 0-1 programming model $\sum_{i=1}^{n} m_{i}$, the model can be solved easily.

2) The calculation is reduced with the concise objective function and constraint conditions.
Owing to the appearance of decision variables in the subscript of constraint conditions, the problem cannot be solved by classical optimization algorithms. Genetic algorithm was adopted in this paper.

1) Given an example, the results of this algorithm is consistent with that by other methods.

2) With further numerical experimentation, when $n, m_{i}(i=1,2, \cdots, n)$ come larger and constraint conditions come more (constraint conditions of 6 indexes were selected multiply), the optimal solution cannot be ensured in Excel. However, the optimal or near optimal solution can be obtained over this algorithm. Thus, the solution can be used in engineering practice.

Therefore, the proposed algorithm is effective.

\section{REFERENCES}

[1] T. J. Lai, "The optimization decision model of sub-contractor selection in multiple subproject," Value Engineering, issue 18, pp. 98-100, 2013.

[2] D. W. Wang, W. X. Ye, and Q. L. Rong, "Enterprise dynamic alliance partner selection model and soft computing," Science in China, vol. 32, no. 6, pp. 824-830, 2002.

[3] D. W. Wang, K. L. Yung, and W. H. Pin, "Partner selection model and soft computing approach for dynamic alliance of enterprises," Science in China, vol. 45, no. 1, pp. 68-80, 2002.

[4] D. W. Wang, Intelligent Optimization Methods, Beijing: Higher Education Press (HEP), April 2007, pp. 19-80.

[5] W. Zhang, J. H. Zhao, and N. Wang, "Integer-coded genetic algorithm with novel repairing mechanism for large scale unit-commitment problem," CIESC Journal, issue 9, pp. 2972-2979, 2012.

[6] E. J. Xia and G. L. Su, "Study on optimization model of bidding assessment on engineering project," China Soft Science, vol. 10, pp. 133-138, 2003.

[7] Q. Zhang, "Project process subcontract management-A new way of enterprise development," Water Conservancy and Hydropower Engineering Cost, vol. 2, pp. 55-57, 2008.

[8] J. S. Zhang, Engineering Project Management, Beijing: Science Press, 2000, pp. 23-68.

[9] The State Development Planning Commission, "The People's Republic of China invite public bidding bids law," The Operation Practice of Tender and Bidding Law, Law Press, 2000.

[10] L. Wang, Intelligent Optimization Method and Its Application, Beijing: Tsinghai University Press, 2001, pp. 36-59.

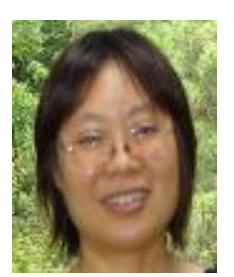

Huiyang Xie was born in Wuxi, Jiangsu province, China on December 18, 1963. She got her bachelor of science degree in mathematics from Anhui Normal University, which is located in Wuhu City, Anhui Province, China, in 1985.

She got her first job in the Department of Mathematics at Anhui Normal University after graduating from the same department in 1985 and served as an assistant lecture from 1985 to 1987 . Then she joined in Anhui Jianzhu University as a lecture in 1989. She moved to Beijing Forestry University in 1993 and severed the university until now as a lecturer, associated professor and became a professor in 2001. She has about 15 publications, include 3 books. Her research interests main focus on applied mathematics, such as financial mathematics, computational algorithms. 